\title{
Betriebswirtschaftliche Kennzahlen
}

Das aktuelle Kapitel vollzieht einen Perspektivwechsel. Wurden FuE- und Innovationsindikatoren bisher vor allem als Blick von außen auf die entsprechenden Einheiten verwendet, soll im Folgenden der Innenblick im Mittelpunkt stehen. Entsprechende Indikatoren können auch für die interne Steuerung von FuE verwendet werden. Auch wenn Projektsteuerung für jeden Sektor wichtig ist, stehen im Weiteren vor allem die Unternehmen im Mittelpunkt. Ihnen stellen sich fünf Fragen, die durch die dann folgenden Indikatoren abgebildet werden:

1. Wie stellt sich (zunächst rein quantitativ) mein FuE-Engagement im Vergleich zum Wettbewerb dar? Hierfür eigenen sich Vergleichsgrößen wie die internen FuE-Aufwendungen in Bezug auf den Umsatz oder die internen FuE-Aufwendungen pro Beschäftigtem in Abhängigkeit von der Unternehmensgröße oder von der Branche (Wissenschaftsstatistik 2019a, Tab. 3.2.3).

Die weiteren Fragen beziehen sich auf einzelne Projekte. Sich immer wieder wiederholende Probleme können aber auch ein Zeichen für einen strukturellen Verbesserungsbedarf sein.

2. Welche Fortschritte macht mein Projekt?

3. Wie ist die Projekteffizienz?

4. Wie entwickeln sich die Kosten?

5. Wie ist die zeitliche Planung?

Mithilfe geeigneter Indikatoren lässt sich ein Controlling-System etablieren, das es der Projekt- und letztlich auch der Unternehmensleitung ermöglicht, Forschung und Innovation so zu gestalten, dass die strategischen Unternehmensziele bestmöglich unterstützt werden. 


\section{Übersicht der Indikatoren}

\section{Bewertung der eigenen FuE- und Innovationsaktivitäten}

9001: FuE-Aufwendungen im Verhältnis zum Umsatz......................................................... 195

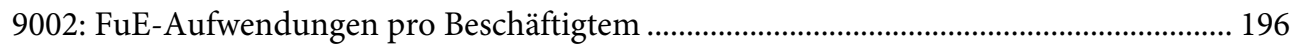

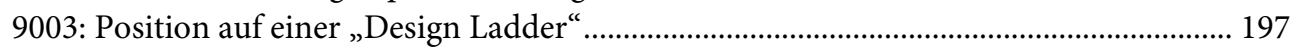

\section{Projektfortschritt}

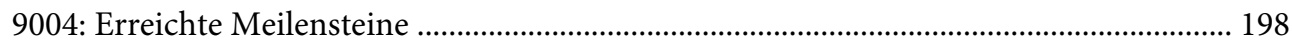

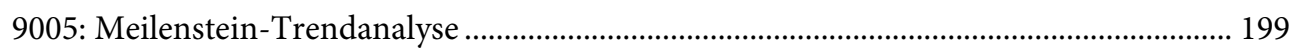

9006: Zahl der abgeschlossenen Arbeitspakete .......................................................................... 200

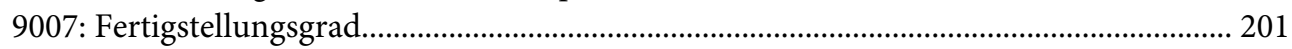

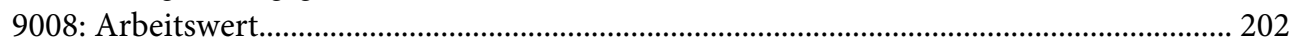

\section{Projekteffizienz}

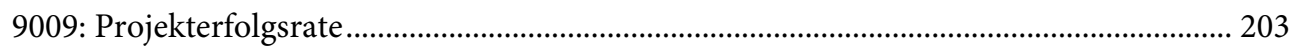

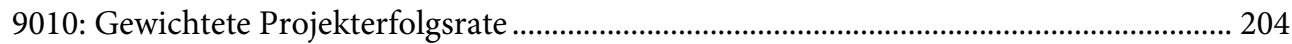

\section{Kosten}

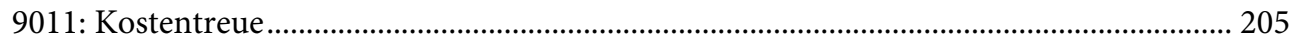

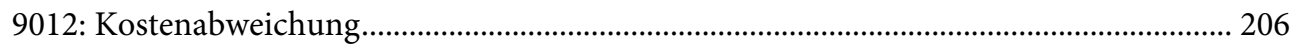

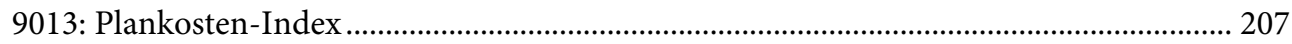

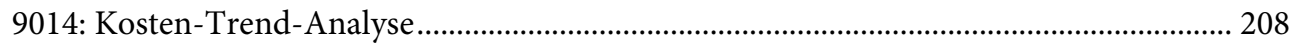

\section{Termine}

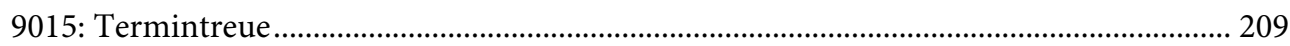

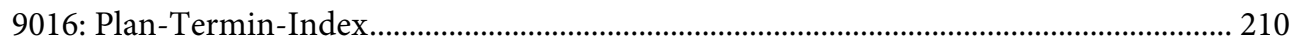

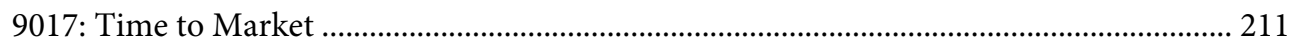




\section{Bewertung der eigenen FuE- und Innovationsaktivitäten}

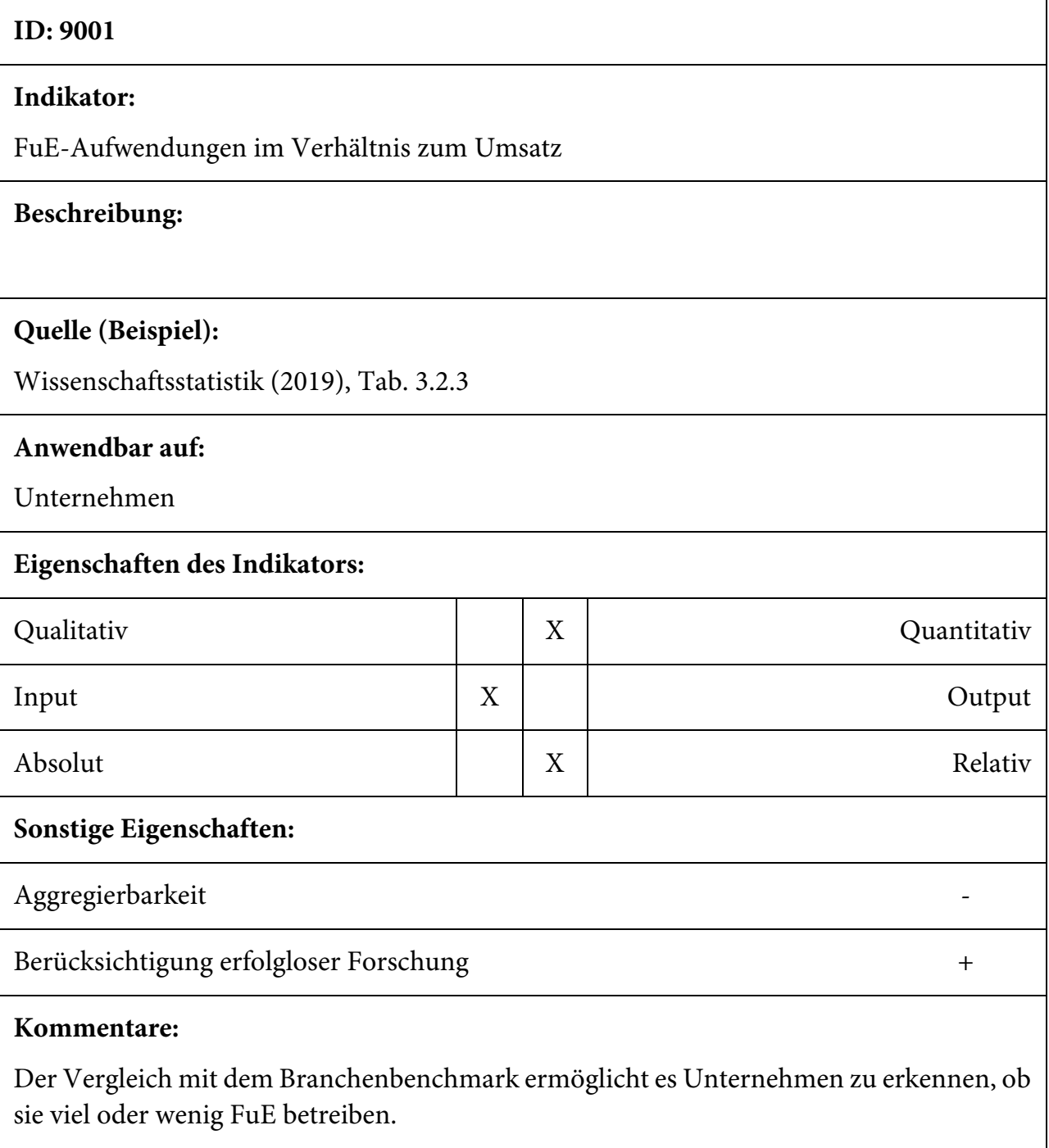




\section{ID: 9002}

\section{Indikator:}

FuE-Aufwendungen pro Beschäftigtem

\section{Beschreibung:}

Quelle (Beispiel):

Wissenschaftsstatistik (2019), Tab. 3.2.3

Anwendbar auf:

Unternehmen

\section{Eigenschaften des Indikators:}

\begin{tabular}{|l|l|l|r|}
\hline Qualitativ & & $\mathrm{X}$ & Quantitativ \\
\hline Input & $\mathrm{X}$ & & Output \\
\hline Absolut & & $\mathrm{X}$ & Relativ \\
\hline
\end{tabular}

Sonstige Eigenschaften:

Aggregierbarkeit

Berücksichtigung erfolgloser Forschung

\section{Kommentare:}

Der Vergleich mit dem Branchenbenchmark ermöglicht es Unternehmen zu erkennen, ob sie viel oder wenig FuE betreiben. 


\section{ID: 9003}

\section{Indikator:}

Position auf einer „Design Ladder“

\section{Beschreibung:}

Position auf einer vierstufigen Skala zur Bedeutung von Design in einem Unternehmen

\section{Quelle (Beispiel):}

OECD (2018), Ziffer 5.99; Galindo-Rueda und Millot (2015); Galindo-Rueda und van Cruysen (2016)

\section{Anwendbar auf:}

Unternehmen

\section{Eigenschaften des Indikators:}

\begin{tabular}{|l|l|l|r|}
\hline Qualitativ & $\mathrm{X}$ & & Quantitativ \\
\hline Input & & $\mathrm{X}$ & Output \\
\hline Absolut & $\mathrm{X}$ & & Relativ \\
\hline
\end{tabular}

\section{Sonstige Eigenschaften:}

Aggregierbarkeit

Berücksichtigung erfolgloser Forschung

\section{Kommentare:}

Design ist nach dem Oslo-Manual (OECD 2018) ein Teil von Innovation, nach dem Frascati-Manual (OECD 2015) aber nur unter sehr eingeschränkten Voraussetzungen ein Teil von FuE. 


\section{Projektfortschritt}

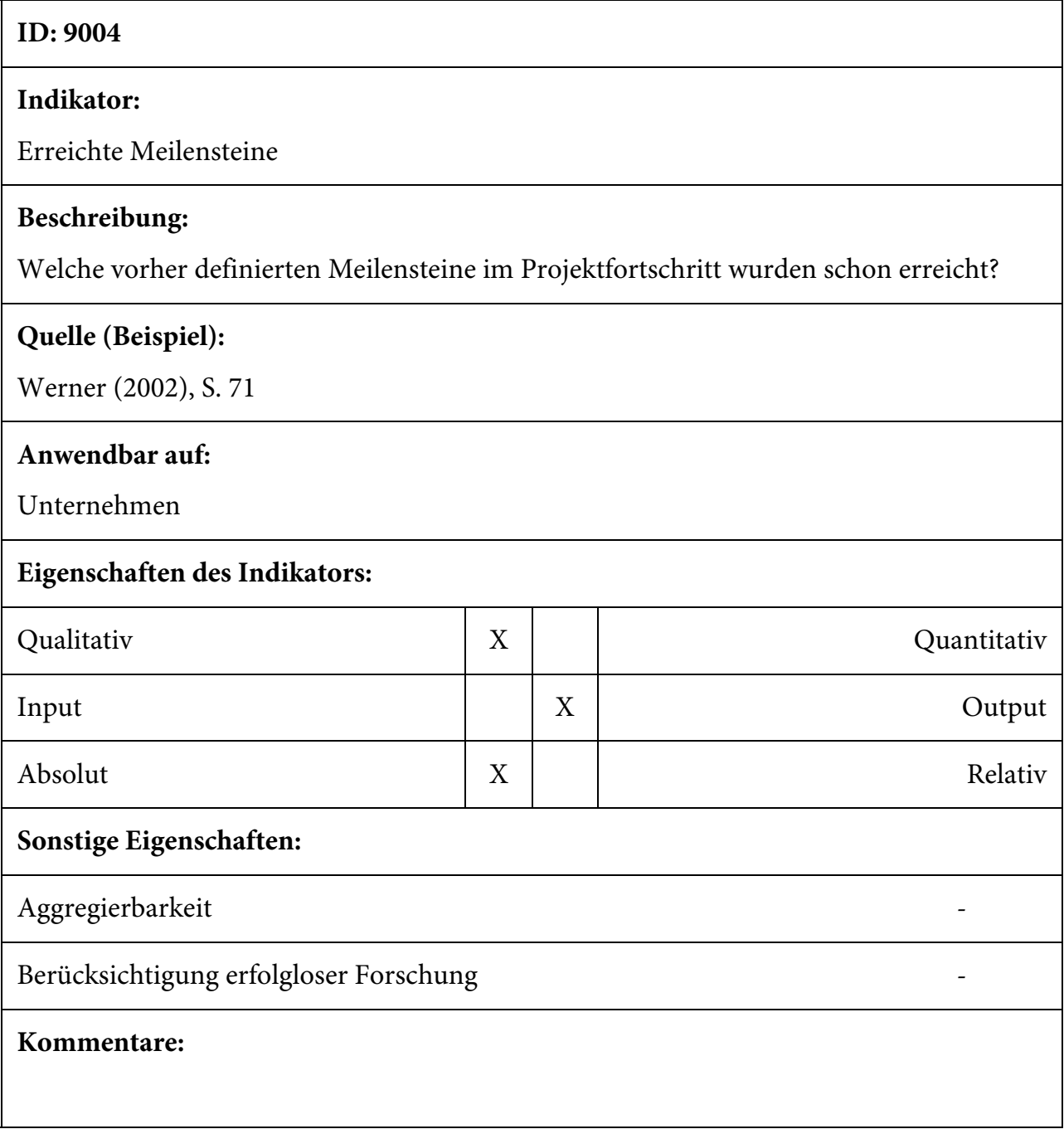




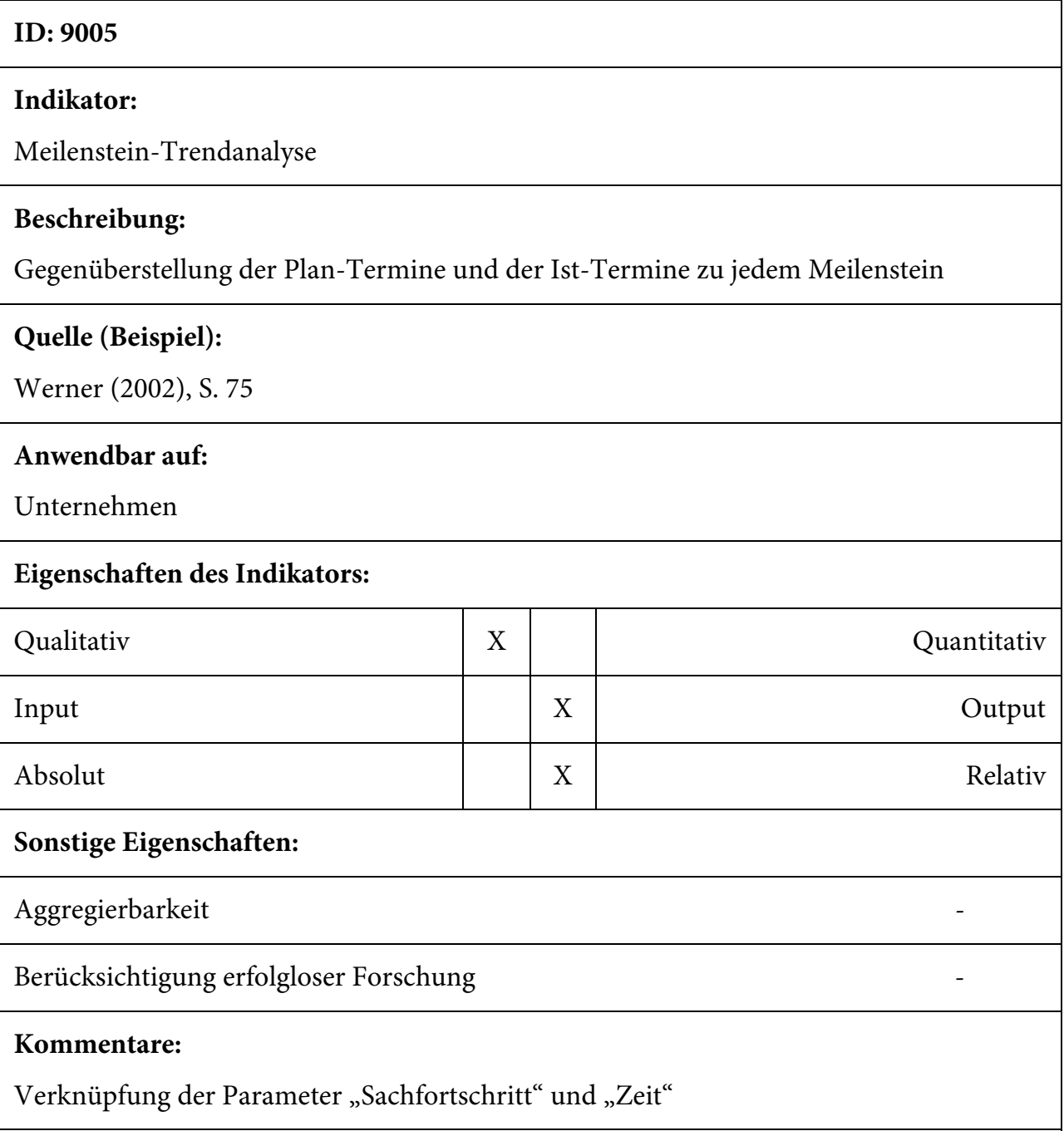




\section{ID: 9006}

\section{Indikator:}

Zahl der abgeschlossenen Arbeitspakete

\section{Beschreibung:}

Bei der Projektplanung werden im Allgemeinen Arbeitspakete definiert. Der Indikator zählt, wie viele davon bereits abgeschlossen wurden.

\section{Quelle (Beispiel):}

Werner (2002), S. 71

\section{Anwendbar auf:}

Unternehmen

\section{Eigenschaften des Indikators:}

\begin{tabular}{|l|l|l|r|}
\hline Qualitativ & & $\mathrm{X}$ & Quantitativ \\
\hline Input & & $\mathrm{X}$ & Output \\
\hline Absolut & $\mathrm{X}$ & & Relativ \\
\hline
\end{tabular}

Sonstige Eigenschaften:

Aggregierbarkeit

Berücksichtigung erfolgloser Forschung

Kommentare: 


\begin{tabular}{|c|c|c|}
\hline \multicolumn{3}{|l|}{ ID: 9007} \\
\hline \multicolumn{3}{|l|}{ Indikator: } \\
\hline \multicolumn{3}{|l|}{ Fertigstellungsgrad } \\
\hline \multicolumn{3}{|l|}{ Beschreibung: } \\
\hline \multicolumn{3}{|c|}{ Zahl der abgeschlossenen Arbeitspakete im Vergleich zur Gesamtzahl der Arbeitspakete } \\
\hline \multicolumn{3}{|l|}{ Quelle (Beispiel): } \\
\hline \multicolumn{3}{|l|}{ Werner (2002), S. 71} \\
\hline \multicolumn{3}{|l|}{ Anwendbar auf: } \\
\hline \multicolumn{3}{|l|}{ Unternehmen } \\
\hline \multicolumn{3}{|l|}{ Eigenschaften des Indikators: } \\
\hline Qualitativ & $\mathrm{X}$ & Quantitativ \\
\hline Input & $\mathrm{X}$ & Output \\
\hline Absolut & $\mathrm{X}$ & Relativ \\
\hline \multicolumn{3}{|l|}{ Sonstige Eigenschaften: } \\
\hline Aggregierbarkeit & & - \\
\hline Berücksichtigung erfolgloser Forschung & & - \\
\hline Kommentare: & & \\
\hline
\end{tabular}




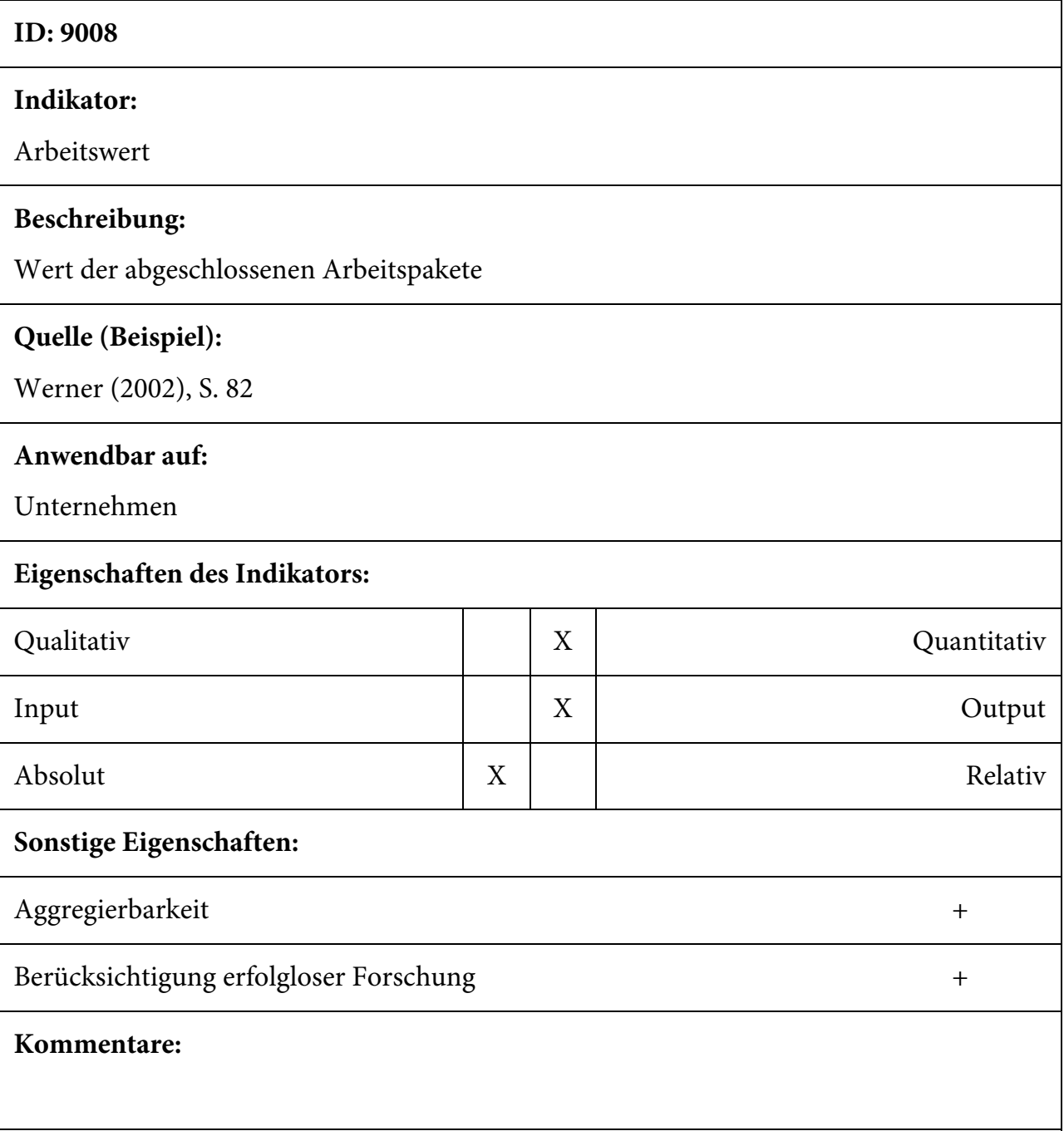




\section{Projekteffizienz}

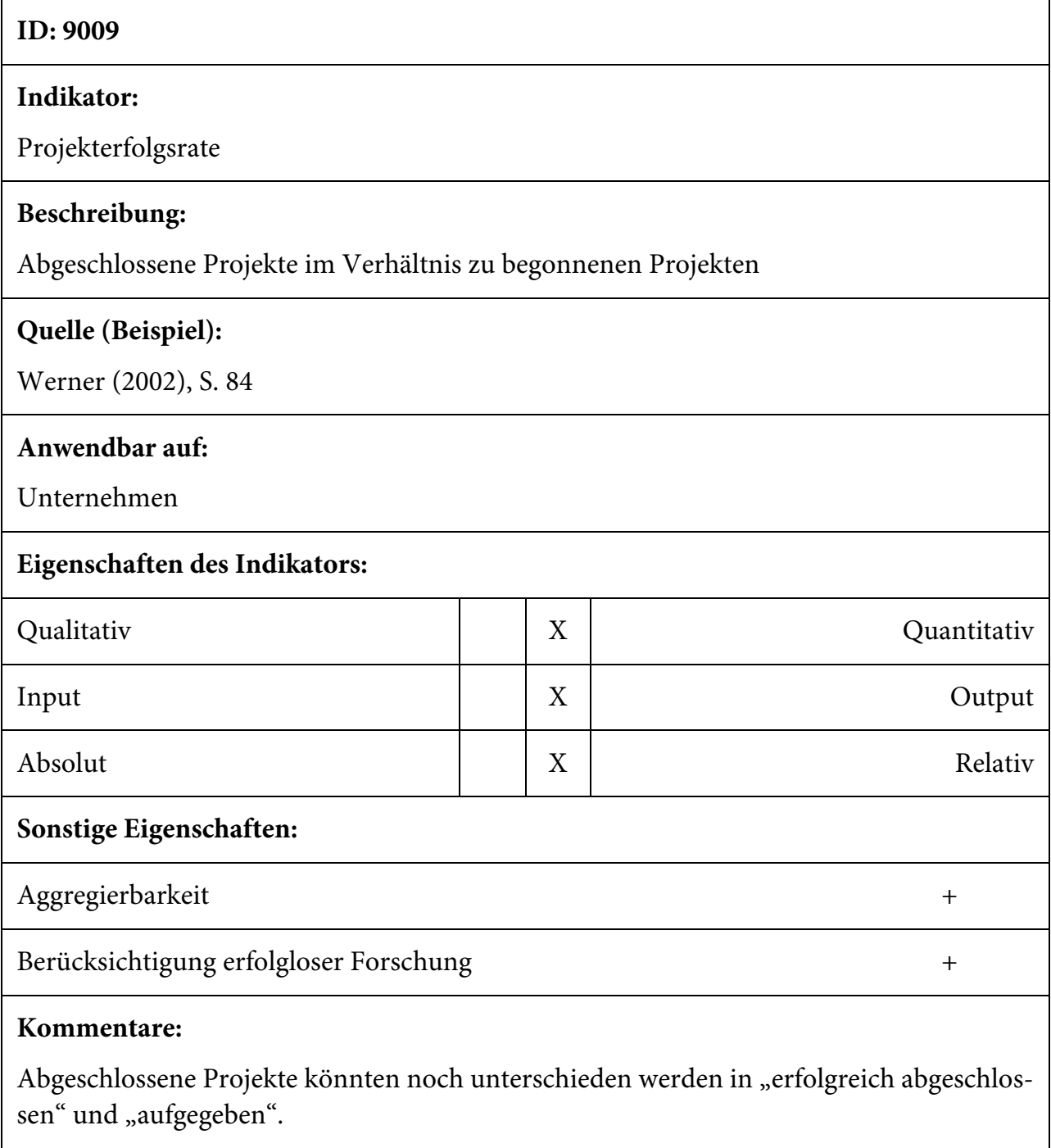




\begin{tabular}{|l|l|l|}
\hline ID: 9010 \\
$\begin{array}{l}\text { Indikator: } \\
\text { Gewichtete Projekterfolgsrate }\end{array}$ \\
\hline $\begin{array}{l}\text { Beschreibung: } \\
\text { Wie Indikator 9009, aber mit den Plankosten gewichtet. }\end{array}$ \\
\hline $\begin{array}{l}\text { Quelle (Beispiel): } \\
\text { Werner (2002), S. 71 }\end{array}$ & $\mathrm{X}$ & \\
\hline Anwendbar auf: & & \\
\hline Unternehmen & $\mathrm{X}$ & \\
\hline Eigenschaften des Indikators: & $\mathrm{X}$ & \\
\hline Qualitativ & & \\
\hline Input & & \\
\hline Absolut & & \\
\hline Sonstige Eigenschaften: & & + \\
\hline Aggregierbarkeit & & \\
\hline Berücksichtigung erfolgloser Forschung & & \\
\hline Kommentare: & & \\
\hline
\end{tabular}




\section{Kosten}

\begin{tabular}{|l|l|l|}
\hline ID: 9011 \\
$\begin{array}{l}\text { Indikator: } \\
\text { Kostentreue }\end{array}$ \\
\hline $\begin{array}{l}\text { Beschreibung: } \\
\text { Istkosten im Verhältnis zu Plankosten }\end{array}$ \\
\hline $\begin{array}{l}\text { Quelle (Beispiel): } \\
\text { Werner (2002), S. 71 }\end{array}$ & & \\
\hline $\begin{array}{l}\text { Anwendbar auf: } \\
\text { Unternehmen }\end{array}$ & $\mathrm{X}$ & \\
\hline Eigenschaften des Indikators: & & \\
\hline Qualitativ & $\mathrm{X}$ & \\
\hline Input & & \\
\hline Absolut & & \\
\hline Sonstige Eigenschaften: & & \\
\hline Aggregierbarkeit & & \\
\hline Berücksichtigung erfolgloser Forschung & & \\
\hline Kommentare: & & \\
\hline
\end{tabular}




\section{ID: 9012}

\section{Indikator:}

Kostenabweichung

Beschreibung:

$$
\frac{\text { Plankosten - Istkosten }}{\text { Plankosten }}
$$

Quelle (Beispiel):

Werner (2002), S. 71

Anwendbar auf:

Unternehmen

Eigenschaften des Indikators:

\begin{tabular}{|l|l|l|r|}
\hline Qualitativ & & $\mathrm{X}$ & Quantitativ \\
\hline Input & $\mathrm{X}$ & & Output \\
\hline Absolut & & $\mathrm{X}$ & Relativ \\
\hline
\end{tabular}

Sonstige Eigenschaften:

Aggregierbarkeit

Berücksichtigung erfolgloser Forschung

Kommentare: 


\section{ID: 9013}

\section{Indikator:}

Plankosten-Index

\section{Beschreibung:}

Neue Plankosten im Vergleich zu alten Plankosten

Quelle (Beispiel):

Werner (2002), S. 73

Anwendbar auf:

Unternehmen

Eigenschaften des Indikators:

\begin{tabular}{|l|l|l|r|}
\hline Qualitativ & & $\mathrm{X}$ & Quantitativ \\
\hline Input & $\mathrm{X}$ & & Output \\
\hline Absolut & & $\mathrm{X}$ & Relativ \\
\hline
\end{tabular}

Sonstige Eigenschaften:

Aggregierbarkeit

Berücksichtigung erfolgloser Forschung

Kommentare:

Nach einer Planrevidierung 


\section{ID: 9014}

\section{Indikator:}

Kosten-Trend-Analyse

\section{Beschreibung:}

Gegenüberstellung der Plankosten und der Istkosten zu jedem Meilenstein

Quelle (Beispiel):

Werner (2002), S. $76 \mathrm{f}$.

Anwendbar auf:

Unternehmen

\section{Eigenschaften des Indikators:}

\begin{tabular}{|c|c|c|c|}
\hline Qualitativ & $\mathrm{X}$ & & Quantitativ \\
\hline Input & & $\mathrm{X}$ & Output \\
\hline Absolut & $\mathrm{X}$ & & Relativ \\
\hline
\end{tabular}

Sonstige Eigenschaften:

Aggregierbarkeit

Berücksichtigung erfolgloser Forschung

\section{Kommentare:}

Verknüpfung der Parameter „Sachfortschritt“ und „Kosten“ 


\section{Termine}

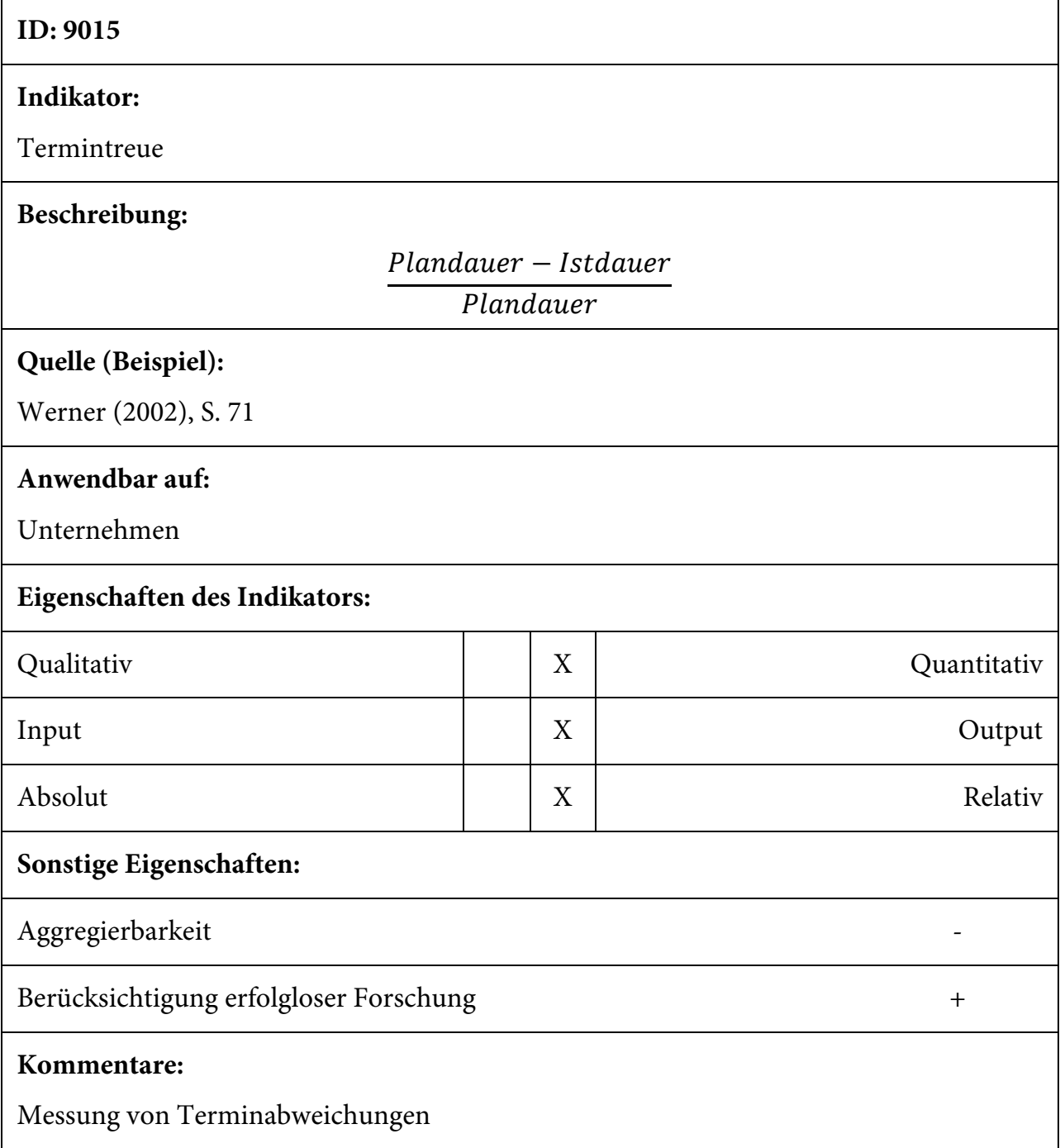




\begin{tabular}{|l|l|l|}
\hline ID: 9016 \\
\hline $\begin{array}{l}\text { Indikator: } \\
\text { Plan-Termin-Index }\end{array}$ \\
\hline $\begin{array}{l}\text { Beschreibung: } \\
\text { Neue Plandauer im Verhältnis zu alter Plandauer }\end{array}$ \\
\hline $\begin{array}{l}\text { Quelle (Beispiel): } \\
\text { Werner (2002), S. 71 }\end{array}$ & $\mathrm{X}$ & \\
\hline $\begin{array}{l}\text { Anwendbar auf: } \\
\text { Unternehmen }\end{array}$ & $\mathrm{X}$ & \\
\hline Eigenschaften des Indikators: & $\mathrm{X}$ & \\
\hline Qualitativ & & \\
\hline Input & & \\
\hline Absolut & & \\
\hline Sonstige Eigenschaften: & & Output \\
\hline Aggregierbarkeit & & \\
\hline Berücksichtigung erfolgloser Forschung & & \\
\hline Kommentare: & & \\
\hline Bei Planrevisionen
\end{tabular}




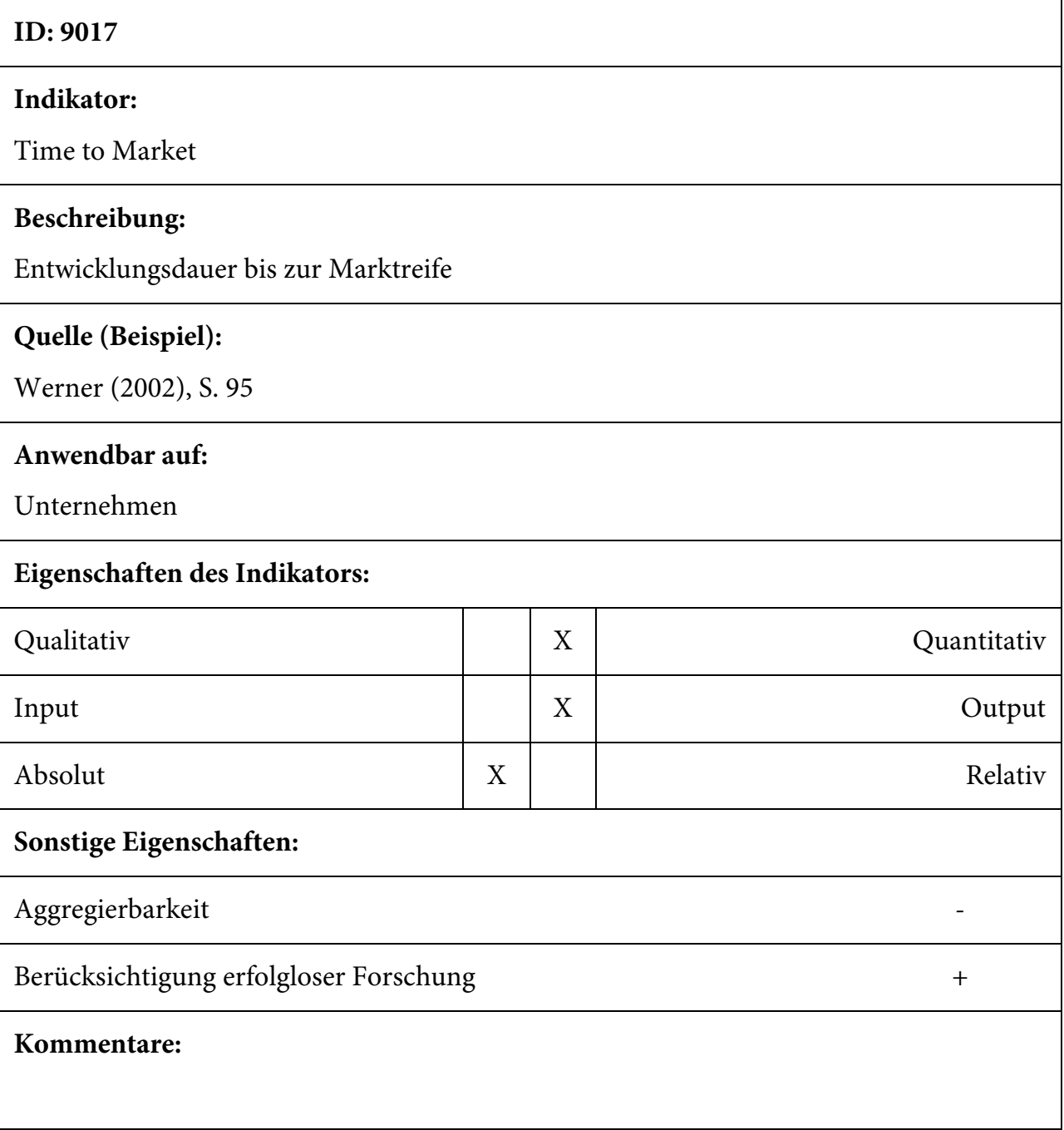




\section{Literatur}

Galindo-Rueda, F., \& Millot, V. (2015). Measuring Design and its Role in Innovation. OECD Science, Technology and Industry Working Papers No. 2015/01. Paris. https://doi.org/10.1787/ 5js7p6lj6zq6-en. Zugegriffen: 18. Sep. 2020

Galindo-Rueda, F., \& van Cruysen, A. (2016). Testing Innovation Survey Concepts, Definitions and Questions: Findings from cognitive Interviews with Business Managers. http://www.oecd.org/ sti/inno/WP_Cognitive\%20Testing\%20of\%20Innovation.pdf. Zugegriffen: 18. Sep. 2020

OECD (2015). Frascati Manual 2015: Guidelines for Collecting and Reporting Data on Research and Experimental Development. Paris: OECD Publishing.

OECD (2018). Oslo Manual 2018: Guidelines for Collecting, Reporting and Using Data on Innovation. Paris: OECD Publishing.

Werner, B. M. (2002). Messung und Bewertung der Leistung von Forschung und Entwicklung im Innovationsprozess. Dissertation. TU Darmstadt. http://tuprints.ulb.tu-darmstadt.de/200/. Zugegriffen: 18. Sep. 2020

Wissenschaftsstatistik (2019). ARENDI Zahlenwerk. Essen. https://stifterverband.org/download/ file/fid/7762. Zugegriffen: 18. Sep. 2020

Open Access Dieses Kapitel wird unter der Creative Commons Namensnennung 4.0 International Lizenz (http://creativecommons.org/licenses/by/4.0/deed.de) veröffentlicht, welche die Nutzung, Vervielfältigung, Bearbeitung, Verbreitung und Wiedergabe in jeglichem Medium und Format erlaubt, sofern Sie den/die ursprünglichen Autor(en) und die Quelle ordnungsgemäß nennen, einen Link zur Creative Commons Lizenz beifügen und angeben, ob Änderungen vorgenommen wurden.

Die in diesem Kapitel enthaltenen Bilder und sonstiges Drittmaterial unterliegen ebenfalls der genannten Creative Commons Lizenz, sofern sich aus der Abbildungslegende nichts anderes ergibt. Sofern das betreffende Material nicht unter der genannten Creative Commons Lizenz steht und die betreffende Handlung nicht nach gesetzlichen Vorschriften erlaubt ist, ist für die oben aufgeführten Weiterverwendungen des Materials die Einwilligung des jeweiligen Rechteinhabers einzuholen.

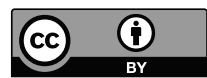

